Pacific Journal of Mathematics

ORTHOGONALLY ADDITIVE AND ORTHOGONALLY
INCREASING FUNCTIONS ON VECTOR SPACES 


\title{
ORTHOGONALLY ADDITIVE AND ORTHOGONALLY INCREASING FUNCTIONS ON VECTOR SPACES
}

\author{
S. Gudder and D. Strawther
}

A real-valued function $f: X \rightarrow R$ on an inner product space $X$ is orthogonally additive if $f(x+y)=f(x)+f(y)$ whenever $x \perp y$. We extend this concept to more general spaces called orthogonality vector spaces. If $X$ is an orthogonality vector space and if there exists an orthogonally additive function on $X$ which satisfies certain natural conditions then there is an inner product on $X$ which is equivalent to the original orthogonality and $f(x)= \pm\|x\|^{2}$ for all $x \in X$. We next consider a normed space $X$ with James' orthogonality. A function $f: X \rightarrow R$ is orthogonally increasing if $f(x+y) \geqq f(x)$ whenever $x \perp y$. Orthogonally increasing functions on normed spaces are characterized.

1. Pythagoras' theorem. Pythagoras' theorem states that the function $f(x)=\|x\|^{2}$ is orthogonally additive, that is $f(x+y)=$ $f(x)+f(y)$ whenever $x \perp y$ where $x, y$ are vectors in the plane. One of the concerns of this paper is a converse of Pythagoras' theorem on an inner product space $X$. That is, if $f: X \rightarrow R$ is orthogonally additive, is $f(x)=c\|x\|^{2}$ for some $c \in R$ ? As it stands, the answer is no, since any linear functional is orthogonally additive.

Some natural additional conditions on $f$ are:

(1) $f(x) \geqq 0$, nonnegativity;

(2) $f(x)=f(-x)$, evenness ;

(3) $\lambda_{i} \rightarrow \lambda$ implies $f\left(\lambda_{i} x\right) \rightarrow f(\lambda x)$ for all $x \in X$, hemicontinuity. We shall show that orthogonal additivity along with (1), or with (2) and (3) imply $f(x)=c\|x\|^{2}$ for some $c \in R$.

2. Orthogonality vector spaces. In this paper, vector spaces will be real and of dimension $\geqq 2$. In Theorem 2.2 we shall prove that Pythagoras' theorem characterizes inner product spaces in a certain sense.

A vector space $X$ is an orthogonality vector space if there is a relation $x \perp y$ on $X$ such that

(01) $x \perp 0,0 \perp x$ for all $x \in X$;

(02) if $x \perp y$ and $x, y \neq 0$, then $x, y$ are linearly independent;

(03) if $x \perp y$, then $a x \perp b y$ for all $a, b \in R$;

(04) if $P$ is a two-dimensional subspace of $X$, then for every $x \in P$ there exists $0 \neq y \in P$ such that $x \perp y$; 
(05) if $P$ is a two-dimensional subspace of $X$, then there exist nonzero vectors $u, v \in P$ such that $u \perp v$ and $u+v \perp u-v$.

Any vector space can be made into an orthogonality vector space if we define $x \perp 0,0 \perp x$ for all $x$, and for nonzero vectors $x, y$ define $x \perp y$ iff $x, y$ are linearly independent. Also an inner product space is such a space; we shall see that a normed space is one also with James' definition of orthogonality.

LEMMA 2.1. Let $(X, \perp)$ be an orthogonality vector space and let $f: X \rightarrow R$ be orthogonally additive and hemi-continuous. (a) If $f$ is odd, then $f$ is linear. (b) If $f$ is even, then $f(\alpha x)=\alpha^{2} f(x)$ for all $\alpha \in R, x \in X$ and if $x \perp y$ and $x+y \perp x-y$, then $f(x)=f(y)$.

Proof. Same as in [2; Lemmas 2, 3].

REMARK. The referee has pointed out to us that there is a mistake in the proof of Lemma 2 [2]. In that proof it is incorrectly stated that $F\left(2^{r} u\right)=2^{r} F(u)$ for all rational $r$ when, in fact, this is only proved for integral $r$. However, it is easily seen that $F\left(3^{r} u\right)=3^{r} F(u)$ for all integral $r$. Indeed, in the notation of that proof

$$
\begin{aligned}
F(3 u)-F(v) & =F(3 u-v)=F(u+v+2 u-2 v) \\
& =F(u+v)+F(2(u-v))=3 F(u)-F(v) .
\end{aligned}
$$

Hence, by induction $F\left(2^{p} 3^{q} u\right)=2^{p} 3^{q} F(u)$ for all integral $p$ and $q$. Since these scalars $2^{p} 3^{q}$ are dense, continuity implies $F(\alpha u)=$ $\alpha F(u)$.

An inner product $\langle\cdot, \cdot\rangle$ on $(X, \perp)$ is $\perp$-equivalent when $x \perp y$ iff $\langle x, y\rangle=0$.

THEOREM 2.2. If there exists an $f:(X, \perp) \rightarrow R$ which is orthogonally additive, even, hemicontinuous, and not identically 0 , then there is a $\perp$-equivalent inner product $\langle\cdot, \cdot\rangle$ on $(X, \perp)$. In fact, $\langle x, y\rangle=$ $\frac{1}{4}[f(x+y)-f(x-y)]$ and the induced norm satisfies $\|x\|^{2}=f(x)$ for all $x \in X$, or $\|x\|^{2}=-f(x)$ for all $x \in X$. Moreover, if $\langle\cdot, \cdot\rangle_{1}$ is another $\perp$-equivalent inner product on $(X, \perp)$, then there is a nonzero $c \in R$ such that $\langle\cdot, \cdot\rangle_{1}=c\langle\cdot, \cdot\rangle$.

Proof. We first show that $f$ has constant sign. Let $0 \neq x \in X$ and suppose $f(x)>0$. Let $0 \neq y \in X$. If $y=\alpha x$, then $f(y)=\alpha^{2} f(x)>$ 0 . If $y, x$ are linearly independent, let $P$ be the generated 2dimensional subspace. Then there exist $u, v \in X$ satisfying (05) and 
(02). Hence $y=a u+b v, x=c u+d v$ for $a, b, c, d \in R$. By Lemma 2.1 (b), $f(y)=\left(a^{2}+b^{2}\right) f(u), f(x)=\left(c^{2}+d^{2}\right) f(u)$ so $f(y)>0$. Similarly, $f(x)<0$ implies $f(y)<0$. For concreteness, suppose $f(x) \geqq 0$ for all $x \in X$. One can now show that $f(x)^{1 / 2}$ is a norm on $X$ which satisfies the parallelogram law so $X$ is an inner product space. If $x \perp y$ then $f(x+y)=f(x)+f(y)$ and so $\langle x, y\rangle=0$. Conversely, suppose $x, y \neq 0$ and $\langle x, y\rangle=0$. By (04) there is a $z \neq 0$ in the span of $\{x, y\}$ such that $x \perp z$. Hence $\langle x, z\rangle=0$ and by (02) $y=a x+b z$ for some $a, b \in R$. From $\langle x, y\rangle=0$ it follows that $a=0$ so $x \perp y$. Corollary 3.4 concludes the proof.

If $X$ is a normed linear space, James [1] defines $x \perp y$ iff $\|x+k y\| \geqq$ $\|x\|$ for all $k \in R$. With this definition of $\perp,(X, \perp)$ is an orthogonality vector space. Indeed, (01), (02), (03) follows easily, (04) follows from [1; Corollary 2.3] and (05) follows from [2; Lemma 1].

The next result generalizes to inner product spaces a result of Sundaresan [2] whose proof relies on the completeness of Hilbert space.

COROLlary 2.3. Let $X$ be a normed space and let $f: X \rightarrow R$ be an orthogonally additive, even, hemicontinuous function. (a) If $X$ is not an inner product space, then $f \equiv 0$. (b) If $X$ is an inner product space, then there is a $c \in R$ such that $f(x)=c\|x\|^{2}$ for all $x \in X$.

We next prove a generalization of the Riesz representation theorem.

Corollary 2.4. Let $X$ be an inner product space and let $f: X \rightarrow R$ be orthogonally additive and satisfy $|f(x)| \leqq M\|x\|$ for all $x \in X$. Then $f$ is a continuous linear functional and hence, if $X$ is a Hilbert space, $f(x)=\langle x, z\rangle$ for some $z \in X$.

Proof. We can assume $M>0$. Clearly $f$ is continuous at 0 . Let $x \neq 0$. We first show that $\beta \rightarrow 1$ implies $f(\beta x) \rightarrow f(x)$. Let $\beta>1$, $y \perp x,\|y\|=1$ and $u=x+(\beta-1)^{1 / 2}\|x\| y$. Then $(u-x) \perp x$ and $(u-\beta x) \perp u$. Thus $f(u)-f(x)=f(u-x)$ and $f(\beta x)-f(u)=$ $f(\beta x-u)$. Hence

$$
\begin{aligned}
|f(x)-f(\beta x)| & \leqq|f(x)-f(u)|+|f(u)-f(\beta x)| \\
& \leqq M\|x\|\left[2(\beta-1)^{1 / 2}+(\beta-1)\right] .
\end{aligned}
$$

Now let $0<\beta<1, y \perp x,\|y\|=1$ and

$$
u=\beta x+(1-\beta)^{1 / 2} \beta^{1 / 2}\|x\| y .
$$


Then $(u-\beta x) \perp \beta x$ and $(x-u) \perp u$. Again $f(u)-f(\beta x)=f(u-\beta x)$, and $f(x)-f(u)=f(x-u)$, so that

$$
\begin{aligned}
|f(x)-f(\beta x)| & \leqq|f(x-u)|+|f(u-\beta x)| \\
& \leqq M\|x\|\left[(1-\beta)+2(1-\beta)^{1 / 2} \beta^{1 / 2}\right] .
\end{aligned}
$$

It follows that $f(\beta x) \rightarrow f(x)$ as $\beta \rightarrow 1$. We now show that $f$ is norm continuous. If $x_{i} \rightarrow x$, there exist $y_{i} \perp x$ such that $x_{i}=$ $\alpha_{i} x+y_{i}$. Taking the inner product with $x$, we see that $\alpha_{i} \rightarrow 1$ and hence $y_{i} \rightarrow 0$. Since $f\left(x_{i}\right)=f\left(\alpha_{i} x+y_{i}\right)=f\left(\alpha_{i} x\right)+f(y)$, we have $f\left(x_{i}\right) \rightarrow f(x)$ as $x_{i} \rightarrow x$ and $f$ is norm continuous. Applying Corollary 2.3 and Lemma 2.1(a), there is a continuous linear functional $f_{2}$ such that $f(x)=c\|x\|^{2}+f_{2}(x)$. Hence $|c|\|x\| \leqq M+\left\|f_{2}\right\|$ for all $x \in X$, which implies $c=0$.

3. Orthogonally increasing functions. In this section orthogonality on a normed space $X$ will always be defined according to James' definition (see §2). A function $f: X \rightarrow R$ is orthogonally increasing iff $x \perp y$ implies $f(x+y) \geqq f(x)$. We shall later define other types of increasing functions.

In the last section we characterized orthogonally additive, hemicontinuous functions. We saw that they formed a very restricted class, being the sum of a linear functional and a constant times the norm squared. The orthogonally increasing functions form a much larger class. Indeed, if $g: R^{+} \rightarrow R$, where $R^{+}=$nonnegative reals, is any nondecreasing function then $f(x)=g(\|x\|)$ is orthogonally increasing since $x \perp y$ implies $f(x+y)=g(\|x+y\|) \geqq g(\|x\|)=f(x)$. The main result of this section characterizes orthogonally increasing functions on a normed space and shows that they are essentially of this form.

Let $X$ be a normed space. A function $f: X \rightarrow R$ is radially increasing if $\alpha>1$ implies $f(\alpha x) \geqq f(x) \forall x \in X$, and $f$ is spherically increasing if $\|x\|>\|y\|$ implies $f(x) \geqq f(y)$. It is clear that spherically increasing implies radially increasing and simple examples show that the converse need not hold. In a strictly convex (rotund) normed space, spherically increasing implies orthogonally increasing. Indeed, let $f$ be a spherically increasing function on such a space and let $x \perp y$. Then $\|x+y\| \geqq\|x\|$. If $\|x+y\|>\|x\|$, then by spherical increasing

$$
f(x+y) \geqq f(x) .
$$

Now suppose $\|x+y\|=\|x\|$. Then

$$
\left\|x+\frac{1}{2} y\right\|=\left\|\frac{1}{2}(x+y)+\frac{1}{2} x\right\| \leqq \frac{1}{2}\|x+y\|+\frac{1}{2}\|x\|=\|x\| .
$$


Since $x \perp y,\left\|x+\frac{1}{2} y\right\| \geqq\|x\|$ so $\left\|x+\frac{1}{2} y\right\|=\|x\|$. But a normed space is strictly convex if and only if $\|u\|=\|v\|=\left\|\frac{1}{2}(u+v)\right\|$ implies $u=v$, and so $\|x+y\|=\|x\|=\left\|x+\frac{1}{2} y\right\|$ implies $y=0$. Hence $f(x+y) \geqq f(x)$ and $f$ is orthogonally increasing. It is well known that any uniformly convex space is strictly convex, in particular an inner product space is strictly convex.

In a general normed space, spherically increasing need not imply orthogonally increasing. Indeed, let $X=\left(R^{2},\|\cdot\|_{\infty}\right)$; that is, $X=R^{2}$ with $\left\|\left(x_{1}, x_{2}\right)\right\|=\max \left(\left|x_{1}\right|,\left|x_{2}\right|\right)$. Note that $X$ is not strictly convex. Let $f: X \rightarrow R$ be defined as follows: $f(x)=\|x\|$ if $0 \leqq\|x\|<1$, $f(x)=2\|x\|$ if $\|x\|>1, f(x)=1$ if $\|x\|=1$ and $x \neq(1,0)$, and $f((1,0))=$ 2. It is easy to check that $f$ is spherically increasing. If $x=(1,0)$ and $y=(0,1)$ then $x \perp y$ but $f(x+y)=f((1,1))=1<2=f(x)$. Hence $f$ is not orthogonally increasing. The next theorem shows that orthogonally increasing implies spherically increasing.

THEOREM 3.1. Let $X$ be a normed space with $\operatorname{dim} X \geqq 2$ and let $f: X \rightarrow R$ be orthogonally increasing. Then $f$ is spherically increasing and there exists a countable number of spheres $S_{1}, S_{2}, \cdots$ such that $f$ is norm continuous at $w$ iff $w \notin \cup S_{i}$. Furthermore, there exists a nondec reasing function $g: R^{+} \rightarrow R$ such that $f(w)=g(\|w\|)$ for every $w \notin \cup S_{i}$.

Proof. We first show that $f$ is radially increasing. Let $0 \neq y \in X$ and let $\alpha>1$. By a modification of the proof of Lemma 1 [2] there exists $0 \neq x \in X$ such that $y \perp x$ and $(y+x) \perp[(\alpha-1) y-x]$. Hence

$$
f(\alpha y)=f[y+x+(\alpha-1) y-x] \geqq f(y+x) \geqq f(y)
$$

and $f$ is radially increasing. We now show that $f$ is norm continuous on a dense subset of $X$. Let $\left\|x_{0}\right\|=1$ and let $V=\left\{\lambda x_{0}: \lambda \in R^{+}\right\}$. Then $f$ restricted to $V$ is an increasing function and hence is continuous in $V$ on a dense subset $B$ of $V$. We shall show that $f$ is norm continuous on $B-\{0\}$. Let $0 \neq x \in B$ and let $x_{i} \rightarrow x$. Now there exists $y_{i}$ such that $x \perp y_{i}$ and $x_{i}=\alpha_{i} x+y_{i}$. Since

$$
\left\|x_{i}-x\right\|=\left\|\left(\alpha_{i}-1\right) x+y_{i}\right\| \geqq\left|\alpha_{i}-1\right|\|x\|
$$

we have $\alpha_{i} \rightarrow 1$. By the Hahn-Banach theorem, there exist continuous linear functionals $f_{x_{i}}$ on $X$ such that $f_{x_{i}}\left(x_{i}\right)=\left\|x_{i}\right\|^{2}$ and $\left\|f_{x_{i}}\right\|=\left\|x_{i}\right\|$. Now

$$
\left|f_{x_{i}}\left(x_{i}\right)-f_{x_{i}}(x)\right|=\left|f_{x_{i}}\left(x_{i}-x\right)\right| \leqq\left\|x_{i}\right\|\left\|x_{i}-x\right\|
$$

so $f_{x_{i}}(x) \rightarrow\|x\|^{2}$. Letting $k_{i}=\left\|x_{i}\right\|^{2} / f_{x_{i}}(x)$ we see that $k_{i} \rightarrow 1$. Furthermore, for every $\alpha \in R$ we have 


$$
\begin{gathered}
\left\|x_{i}+\alpha\left(k_{i} x-x_{i}\right)\right\| \geqq f_{x_{i}}\left[(1-\alpha) x_{i}+\alpha k_{i} x\right] /\left\|f_{x_{i}}\right\| \\
=\left[(1-\alpha)\left\|x_{i}\right\|^{2}+\alpha k_{i} f_{x_{i}}(x)\right] /\left\|f_{x_{i}}\right\|=\left\|x_{i}\right\| .
\end{gathered}
$$

Hence $x_{i} \perp\left(k_{i} x-x_{i}\right)$. Thus

$$
f\left(k_{i} x\right)=f\left(x_{i}+k_{i} x-x_{i}\right) \geqq f\left(x_{i}\right)=f\left(\alpha_{i} x+y_{i}\right) \geqq f\left(\alpha_{i} x\right) .
$$

Since $\alpha_{i}, k_{i} \rightarrow 1$ we have $f\left(k_{i} x\right), f\left(\alpha_{i} x\right) \rightarrow f(x)$ so $f\left(x_{i}\right) \rightarrow f(x)$ and $f$ is norm continuous on a dense subset of $X$. We next show that $f$ is spherically increasing. Let $x, y \in X$ and suppose $\|y\|>\|x\|$. We shall show there exists $\lambda>1$ and $x=x_{0}, x_{1}, \cdots, x_{n} \in X$ such that $y=\lambda x_{n}$ and $x_{i-1} \perp\left(x_{i}-x_{i-1}\right), i=1, \cdots, n$. It would then follow that

$$
f(y)=f\left(\lambda x_{n}\right) \geqq f\left(x_{n}\right)=f\left(x_{n-1}+x_{n}-x_{n-1}\right) \geqq f\left(x_{n-1}\right) \geqq \cdots \geqq f\left(x_{0}\right)=f(x) .
$$

To show such $\lambda$ and $x_{i}$ exist we proceed as follows. We can assume without loss of generality that $\|x\|=1$, that $x$ and $y$ are linearly independent, and that the 2-dimensional subspace generated by $\{x, y\}$ is $R^{2}$ with $x=(1,0)$. Let $S$ be the unit sphere in $R^{2}$ corresponding to the unit sphere in $X$. Since the norm is a convex function, using polar coordinates, we can assume that $S$ is given by $\rho=F(\theta)$ where $F$ is a continuous function on $[0,2 \pi]$, which is periodic of period $\pi$, the right-hand derivative $F^{\prime}$ exists everywhere, and $F^{\prime}$ is bounded. Let $S_{0}$ be a unit sphere obtained by reflecting $S$ about the $x$-axis. Then, in polar coordinates, $S_{0}$ is given by $\rho_{0}=F_{0}(\theta)$ where $F_{0}(\theta)=$ $F(2 \pi-\theta)$. Denote orthogonality with respect to $S$ and $S_{0}$ by $\perp$ and $\perp_{0}$ respectively, and the norm with respect to $S$ and $S_{0}$ by $\|\cdot\|$ and $\|\cdot\|_{0}$ respectively. We now construct a polygonal path $P$ starting at $x$ and sweeping twice around the origin with vertices $x_{0}=x, x_{1}, x_{2}, \cdots, x_{2 n}$ as follows. The angle between $x_{i-1}$ and $x_{i}$ is $2 \pi / n, x_{i-1} \perp\left(x_{i}-x_{i-1}\right)$ for $i=1,2, \cdots, n$, and $x_{i-1} \perp_{0}\left(x_{i}-x_{i-1}\right)$ for $i=n+1, n+2, \cdots, 2 n$. Now

$$
\left\|x_{2 n}\right\|_{0} \geqq\left\|x_{2 n-1}\right\|_{0} \geqq \cdots \geqq\left\|x_{n}\right\|_{0}=\left\|x_{n}\right\| \geqq\left\|x_{n-1}\right\| \geqq \cdots \geqq\|x\| \text {. }
$$

Indeed, since $x_{2 n}=x_{2 n-1}+\left(x_{2 n}-x_{2 n-1}\right)$ we have $\left\|x_{2 n}\right\|_{0} \geqq\left\|x_{2 n-1}\right\|_{0}$ and the others follow in a similar way. Furthermore, $\left\|x_{n}\right\| \geqq\|w\|$ for any $w \in P$ which precedes $x_{n}$. Indeed, if $w$ is on the edge with vertices $x_{n}$ and $x_{n-1}$ then $w=\lambda x_{n}+(1-\lambda) x_{n-1}$ for some $0 \leqq \lambda \leqq 1$ and hence $\|w\| \leqq$ $\lambda\left\|x_{n}\right\|+(1-\lambda)\left\|x_{n-1}\right\| \leqq\left\|x_{n}\right\|$. A similar argument holds for other $w \in$ $P$. Hence, if we can show that $\lim _{n \rightarrow \infty}\left\|x_{2 n}\right\|_{0}=1$ we will be finished with this part of the proof. A simple calculation shows that the slope of $S$ in the forward direction at angle $\theta$ is 
Since $x \perp\left(x_{1}-x\right)$ it follows that the slope of $x_{1}-x$ equals the slope of $S$ in the forward direction at $\theta=0$. Letting $\rho_{1}$ be the $\rho$ coordinate of $x_{1}$ we have

$$
\rho_{1} \sin (2 \pi / n) /\left[\rho_{1} \cos (2 \pi / n)-1\right]=\left[F^{\prime}(0)\right]^{-1} .
$$

Hence

$$
\rho_{1}=\left[\cos (2 \pi / n)-F^{\prime}(0) \sin (2 \pi / n)\right]^{-1}
$$

and this formula holds even if $F^{\prime}(0)=0$. In a similar way, a straightforward calculation gives

$$
\rho_{i}=\rho_{i-1}\left\{\cos (2 \pi / n)-\left[F^{\prime}(2 \pi i / n) / F(2 \pi i / n)\right] \sin (2 \pi / n)\right\}^{-1},
$$

$i=2,3, \cdots, n$. A similar formula holds for $\rho_{0 i}, \quad i=n+1$, $n+2, \cdots, 2 n$. Using the fact that $F_{0}(2 \pi i / n)=F[2 \pi(n-i) / n]$ and $F_{0}^{\prime}(2 \pi i / n)=-F^{\prime}[2 \pi(n-i) / n]$ we obtain

$$
\begin{aligned}
\rho_{02 n}= & \left\{\cos ^{2}(2 \pi / n)-\left[F^{\prime}(0)\right]^{2} \sin ^{2}(2 \pi / n)\right\}^{-1} \\
& \times\left\{\cos ^{2}(2 \pi / n)-\left[F^{\prime}(2 \pi / n) / F(2 \pi / n)\right]^{2} \sin ^{2}(2 \pi / n)\right\}^{-1} \\
& \times \cdots \times\left\{\cos ^{2}(2 \pi / n)-\left[F^{\prime}((n-1) 2 \pi / n) / F((n\right.\right. \\
& \left.\quad-1) 2 \pi / n)]^{2} \sin ^{2}(2 \pi / n)\right\}^{-1} .
\end{aligned}
$$

Letting $M=\sup \left[F^{\prime}(\theta) / F(\theta)\right]^{2}$ we have

$$
\lim _{n \rightarrow \infty} \rho_{02 n} \leqq \lim _{n \rightarrow \infty}\left[\cos ^{2}(2 \pi / n)-M \sin ^{2}(2 \pi / n)\right]^{-n}
$$

But L'Hospital's rule shows that

$$
\lim _{x \rightarrow 0}(2 \pi / x) \log \left[\cos ^{2} x-M \sin ^{2} x\right]=0
$$

so

$$
\lim _{n \rightarrow \infty} \rho_{02 n}=1 \text {. Hence } \lim _{n \rightarrow \infty}\left\|x_{2 n}\right\|_{0}=1
$$

We next show that $f$ is norm continuous except on a countable set of spheres. Let $\left\|x_{0}\right\|=1$. Then from the above, $f$ is norm continuous at $\delta x_{0}$ except for countably many $\delta$ 's, say $\delta_{1}, \delta_{2}, \cdots$. Suppose $f$ is continuous at $x=\delta x_{0}$ and $\|y\|=\|x\|$. If $\lambda>1$ then $f(\lambda x) \geqq f(y)$, so 
letting $\lambda \rightarrow 1$ we have $f(x) \geqq f(y)$ and in a similar way we show that $f(x) \geqq f(y)$ so $f(x)=f(y)$. To show $f$ is continuous at $y$, let $y_{i} \rightarrow y$. As $\|y\|=\|x\|>0$, it is possible, for $i$ sufficiently large, to find a sequence $a_{i} \in R$ such that $a_{i} \rightarrow 0, a_{i}>0$ and $\left\|y_{i}\right\|-a_{i}>0$. Let $x_{i}=$ $\left(\left\|y_{i}\right\|+a_{i}\right) x /\|y\|$ and $z_{i}=\left(\left\|y_{i}\right\|-a_{i}\right) x /\|y\|$. Then $\left\|x_{i}\right\|>\left\|y_{i}\right\|>\left\|z_{i}\right\|$ so $f\left(z_{i}\right) \leqq f\left(y_{i}\right) \leqq f\left(x_{i}\right)$. Now $x_{i} \rightarrow x, z_{i} \rightarrow x$ and since $f$ is continuous at $x$ we have $f\left(y_{i}\right) \rightarrow f(x)=f(y)$. Hence $f$ is continuous at $y$. If $S_{i}=$ $\left\{x \in X ;\|x\|=\delta_{i}\right\}$, it follows that $f$ is continuous at $w$ iff $w \notin U$ $S_{i}$. Define $g: R^{+} \rightarrow R$ by $g(\alpha)=f\left(\alpha x_{0}\right)$. Then $g$ is a nondecreasing function and if $w \notin \cup S_{i}$ we have $f(w)=f\left(\|w\| x_{0}\right)=g(\|w\|)$.

Using Theorem 3.1 we can prove a result similar to Corollary 2.3 concerning nonnegative orthogonally additive functions.

COROllary 3.2. Let $X$ be a normed space with $\operatorname{dim} X \geqq 2$ and let $f: X \rightarrow R^{+}$be orthogonally additive. (a) If $X$ is not an inner product space, then $f \equiv 0$. (b) If $X$ is an inner product space, then there is a $c \in R^{+}$such that $f(x)=c\|x\|^{2}$ for all $x \in X$.

In the rest of this section $X$ will denote an inner product space with $\operatorname{dim} X \geqq 2$ and inner product $\langle\cdot, \cdot\rangle$.

Corollary 3.3. If $f: X \rightarrow R^{+}$is orthogonally additive, then there is a $c \in R^{+}$with $f(x)=c\|x\|^{2}$.

Corollary 3.4. Let $\langle\cdot, \cdot\rangle_{1}$ be another inner product on $X$. If $x \perp y$ implies $x \perp_{1} y$, then there is a $c>0$ such that $\langle u, v\rangle_{1}=c\langle u, v\rangle$ for all $u, v \in X$.

Proof. Let $g(w)=\|w\|_{1}$. If $x \perp y$ then $x \perp_{1} y$ so $g^{2}(x+y)=$ $g^{2}(x)+g^{2}(y)$. Hence $g^{2}$ is orthogonally additive so there is a $c>0$ with $\|w\|_{1}=g(w)=c\|w\|$. Hence

$$
\begin{aligned}
\langle u, v\rangle_{1} & =\left[\|u+v\|_{1}^{2}-\|u-v\|_{1}^{2}\right] / 4=c^{2}\left[\|u+v\|^{2}-\|u-v\|^{2}\right] / 4 \\
& =c^{2}\langle u, v\rangle .
\end{aligned}
$$

CoRollary 3.5. If $f: X \rightarrow R$ is orthogonally additive and $f(x) \geqq$ $-M\|x\|^{2}$ for all $x \in X$ for some $M \geqq 0$, then there is an $\alpha \in R$ such that $f(x)=\alpha\|x\|^{2}$.

Proof. If $g(x)=f(x)+M\|x\|^{2}$, then $g: X \rightarrow R^{+}$is orthogonally additive. Hence there is a $c \geqq 0$ such that $g(x)=c\|x\|^{2}$. Hence $f(x)=(c-M)\|x\|^{2}$.

In a similar way, Corollary 3.5 holds if $f(x) \leqq M\|x\|^{2}$, for all $x \in X$. 
Let $x_{0} \in X, c, d \in R^{+}$and define $f(x)=c\left\|x-x_{0}\right\|^{2}+d$. Then $f(x) \geqq f\left(x_{0}\right)$ and if $x \perp y$ we have

$$
\begin{aligned}
f(x+y) & =c\left\|x-x_{0}\right\|^{2}-2 c\left\langle y, x_{0}\right\rangle+c\|y\|^{2}+d \\
& =c\left\|x-x_{0}\right\|^{2}+c\left\|y-x_{0}\right\|^{2}-c\left\|x_{0}\right\|^{2}+d \\
& =f(x)+f(y)-d-c\left\|x_{0}\right\|^{2}=f(x)+f(y)-f(0) .
\end{aligned}
$$

We now show that the converse holds.

Corollary 3.6. Let $f: X \rightarrow R$ satisfy: (a) there is an $x_{0} \in X$ such that $f(x) \geqq f\left(x_{0}\right)$ for all $x \in X$, (b) if $x \perp y$ then

$$
f(x+y)=f(x)+f(y)-f(0) .
$$

Then is a $c \geqq 0$ such that $f(x)=c\left\|x-x_{0}\right\|^{2}+f\left(x_{0}\right)$ and if $c \neq 0, x_{0}$ is unique.

Proof. Let $g(x)=f\left(x+x_{0}\right)-f\left(x_{0}\right)$. Then $g: X \rightarrow R^{+}$. Let $x \perp y$ and write $x=x_{1}+x_{2}+x_{3}$ where $x_{1}$ is a multiple of $x, x_{2}$ is a multiple of $y$ and $x_{3}$ is orthogonal to $x$ and $y$. Then $g(x+y)=g(x)+g(y)$. Hence $g(x)=c\|x\|^{2}$ for some $c \geqq 0$ and $f\left(x+x_{0}\right)=c\|x\|^{2}+f\left(x_{0}\right)$. Hence $f(x)=c\left\|x-x_{0}\right\|^{2}+f\left(x_{0}\right)$. If $c \neq 0$ and $f(x) \geqq f\left(y_{0}\right)$ for all $x \in X$ then $f\left(y_{0}\right)=f\left(x_{0}\right)$ and $f\left(y_{0}\right)=c\left\|y_{0}-x_{0}\right\|^{2}+f\left(x_{0}\right)$. Thus $\left\|y_{0}-x_{0}\right\|=0$ so $y_{0}=$ $x_{0}$.

COROllary 3.7. Let $f: X \rightarrow R$ be orthogonally additive. If there is an $x_{0} \in X$ such that $f\left(x_{0}\right)=\left\|x_{0}\right\|^{2}$ and $|f(x)| \leqq\left\|x_{0}\right\|\|x\|$ for all $x \in X$, then $f(x)=\left\langle x, x_{0}\right\rangle$ for all $x \in X$.

Proof. Let $g(x)=\|x\|^{2}-2 f(x)+\left\|x_{0}\right\|^{2}$. Then

$$
g(x) \geqq\|x\|^{2}-2\|x\|\left\|x_{0}\right\|+\left\|x_{0}\right\|^{2}=\left(\|x\|+\left\|x_{0}\right\|\right)^{2} \geqq 0=g\left(x_{0}\right) .
$$

Also $x \perp y$ implies $g(x+y)=g(x)+g(y)-g(0)$. Hence by Corollary 3.6 there is a $c \geqq 0$ such that $g(x)=c\left\|x-x_{0}\right\|^{2}$. Therefore

$$
\begin{aligned}
2 f(x)= & \|x\|^{2}+\left\|x_{0}\right\|^{2}-c\left\|x-x_{0}\right\|^{2}=(1-c)\|x\|^{2} \\
& +(1-c)\left\|x_{0}\right\|^{2}+2 c\left\langle x, x_{0}\right\rangle .
\end{aligned}
$$

Since $f(0)=0$ we have $(1-c)\left\|x_{0}\right\|^{2}=0$. Thus either $c=1$ or $x_{0}=0$. If $x_{0}=0$ then $|1-c|\|x\|^{2}=2|f(x)| \leqq 0$ for all $x \in X$ so again $c=1$. Hence $f(x)=\left\langle x, x_{0}\right\rangle$. 
ACKNOWLedgment. The authors would like to thank the referee whose comments helped to improve this paper and generalize some of the results.

\section{REFERENCES}

1. R. C. James, Orthogonality and linear functionals in normed linear spaces, Trans. Amer. Math. Soc., 61 (1947), 265-292.

2. K. Sundaresan, Orthogonality and nonlinear functionals on Banach spaces, Proc. Amer. Math. Soc., 34 (1972), 187-190.

Received March 28, 1974 and in revised form December 26, 1974.

UNIVERSITY OF DENVER 


\section{PACIFIC JOURNAL OF MATHEMATICS}

\section{EDITORS}

RICHARD ARENS (Managing Editor)

University of California

Los Angeles, California 90024

\section{J. DugundJI}

Department of Mathematics University of Southern California Los Angeles, California 90007

D. Gilbarg and J. Milgram

Stanford University

Stanford, California 94305

\section{ASSOCIATE EDITORS}
E. F. BECKENBACH
B. H. NeumanN
F. WoLF
K. YoshiDA

\section{SUPPORTING INSTITUTIONS}

\author{
UNIVERSITY OF BRITISH COLUMBIA \\ CALIFORNIA INSTITUTE OF TECHNOLOGY \\ UNIVERSITY OF CALIFORNIA \\ MONTANA STATE UNIVERSITY \\ UNIVERSITY OF NEVADA \\ NEW MEXICO STATE UNIVERSITY \\ OREGON STATE UNIVERSITY \\ UNIVERSITY OF OREGON \\ OSAKA UNIVERSITY
}

\author{
UNIVERSITY OF SOUTHERN CALIFORNIA \\ STANFORD UNIVERSITY \\ UNIVERSITY OF TOKYO \\ UNIVERSITY OF UTAH \\ WASHINGTON STATE UNIVERSITY \\ UNIVERSITY OF WASHINGTON \\ AMERICAN MATHEMATICAL SOCIETY
}

The Supporting Institutions listed above contribute to the cost of publication of this Journal, but they are not owners or publishers and have no responsibility for its contents or policies.

Mathematical papers intended for publication in the Pacific Journal of Mathematics should be in typed form or offset-reproduced (not dittoed), double spaced with large margins. Underline Greek letters in red, German in green, and script in blue. The first paragraph or two must be capable of being used separately as a synopsis of the entire paper. Items of the bibliography should not be cited there unless absolutely necessary, in which case they must be identified by author and Journal, rather than by item number. Manuscripts, in duplicate, may be sent to any one of the four editors. Please classify according to the scheme of Math. Reviews, Index to Vol. 39. All other communications should be addressed to the managing editor, or Elaine Barth, University of California, Los Angeles, California, 90024.

100 reprints are provided free for each article, only if page charges have been substantially paid. Additional copies may be obtained at cost in multiples of 50 .

The Pacific Journal of Mathematics is issued monthly as of January 1966. Regular subscription rate: $\$ 72.00$ a year (6 Vols., 12 issues). Special rate: $\$ 36.00$ a year to individual members of supporting institutions.

Subscriptions, orders for back numbers, and changes of address should be sent to Pacific Journal of Mathematics, 103 Highland Boulevard, Berkeley, California, 94708.

PUBLISHED BY PACIFIC JOURNAL OF MATHEMATICS, A NON-PROFIT CORPORATION Printed at Jerusalem Academic Press, POB 2390, Jerusalem, Israel.

$$
\begin{gathered}
\text { Copyright (C) } 1975 \text { Pacific Journal of Mathematics } \\
\text { All Rights Reserved }
\end{gathered}
$$




\section{Pacific Journal of Mathematics}

\section{Vol. 58, No. $2 \quad$ April, 1975}

Zvi Artstein and John Allen Burns, Integration of compact set-valued functions . . . . . . . . . 297

Mark Benard, Characters and Schur indices of the unitary reflection group $[321]^{3} \ldots \ldots \ldots . .309$

Simeon M. Berman, A new characterization of characteristic functions of absolutely continuous

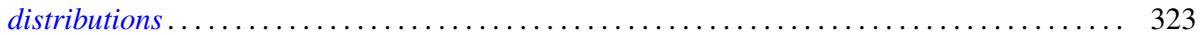

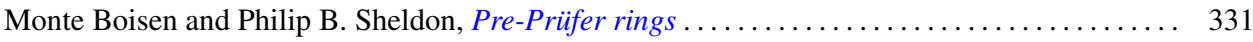

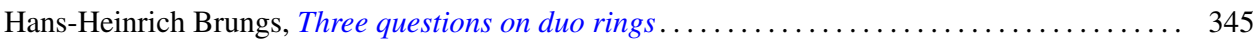

Iracema M. Bund, Birnbaum-Orlicz spaces of functions on groups................. 351

John D. Elwin and Donald R. Short, Branched immersions between 2-manifolds of higher

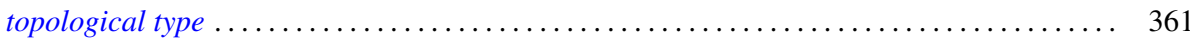

Eric Friedlander, Extension functions for rank 2, torsion free abelian groups . .......... 371

Jon Froemke and Robert Willis Quackenbush, The spectrum of an equational class of

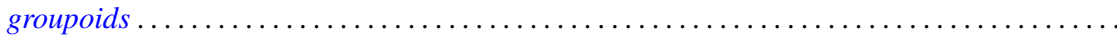

Barry J. Gardner, Radicals of supplementary semilattice sums of associative rings ...........

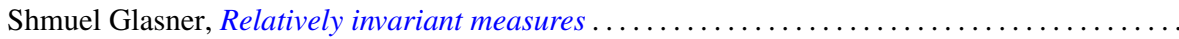

George Rudolph Gordh, Jr. and Sibe Mardesic, Characterizing local connectedness in inverse

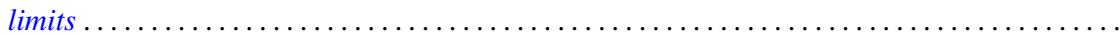

Siegfried Graf, On the existence of strong liftings in second countable topological spaces......

Stanley P. Gudder and D. Strawther, Orthogonally additive and orthogonally increasing

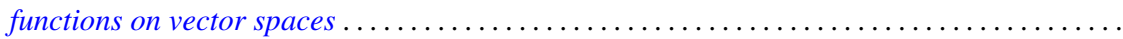

Darald Joe Hartfiel and Carlton James Maxson, A characterization of the maximal monoids and

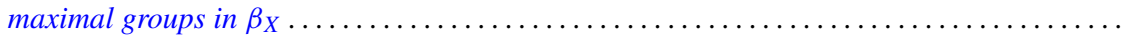

Robert E. Hartwig and S. Brent Morris, The universal flip matrix and the generalized faro-shuffle. .

William Emery Haver, Mappings between ANRs that are fine homotopy equivalences. .

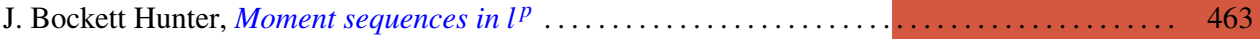

Barbara Jeffcott and William Thomas Spears, Semimodularity in the completion of a poset.... 467

Jerry Alan Johnson, A note on Banach spaces of Lipschitz functions . . . . . . . . . . . . 475

David W. Jonah and Bertram Manuel Schreiber, Transitive affine transformations on

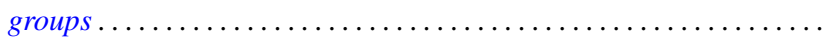

Karsten Juul, Some three-point subset properties connected with Menger's characterization of

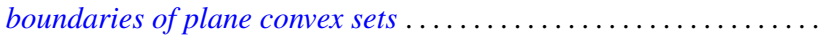

Ronald Brian Kirk, The Haar integral via non-standard analysis . . . . . . . . . . . . . 517

Justin Thomas Lloyd and William Smiley, On the group of permutations with countable

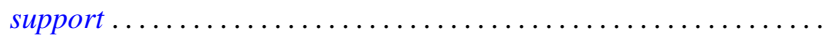

Erwin Lutwak, Dual mixed volumes .................................. 531

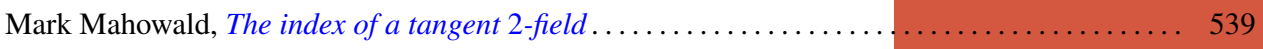

Keith Miller, Logarithmic convexity results for holomorphic semigroups . . . . . . . . . . . . 549

Paul Milnes, Extension of continuous functions on topological semigroups . . . . . . . . . . 553

Kenneth Clayton Pietz, Cauchy transforms and characteristic functions ................ 563

James Ted Rogers Jr., Whitney continua in the hyperspace $C(X) \ldots \ldots \ldots \ldots \ldots \ldots \ldots \ldots .569$

Jean-Marie G. Rolin, The inverse of a continuous additive functional . . . . . . . . . . . . 585

William Henry Ruckle, Absolutely divergent series and isomorphism of subspaces . ........ 605

Rolf Schneider, A measure of convexity for compact sets . ..................... 617

Alan Henry Schoenfeld, Continous measure-preserving maps onto Peano spaces .......... 627

V. Merriline Smith, Strongly superficial elements .......................... 643

Roger P. Ware, A note on quadratic forms over Pythagorean fields . . . . . . . . . . . . . . 651

Roger Allen Wiegand and Sylvia Wiegand, Finitely generated modules over Bezout rings . . . . 655

Martin Ziegler, A counterexample in the theory of definable automorphisms . . . . . . . . . 665 